\title{
Percepção do envelhecimento: mulheres de meia idade e idosas que buscam por procedimentos estéticos
}

\author{
Flávia Franco Carrara ${ }^{1}$; Carmen Guilherme Christiano de Matos Vinagre ${ }^{2}$; Luciane Lúcio Pereira ${ }^{3}$
}

\begin{abstract}
Resumo: Este estudo teve como objetivo: analisar se há diferença estatística significativa relativa à percepção do envelhecimento entre mulheres de meia idade e mulheres idosas que buscam por procedimentos estéticos e à percepção da melhora da saúde com a realização de procedimentos estéticos neste mesmo grupo. Método: Estudo clínico, piloto, observacional e transversal, realizado com 70 mulheres de 40 anos a 75 anos de idade, adeptas de procedimentos estéticos, na cidade de São Paulo (SP), Brasil. Os instrumentos utilizados foram: Questionário de Percepção do Envelhecimento validado e Questionários sociodemográfico e de caracterização das participantes. Para a análise dos dados foram utilizados os Testes Qui-quadrado, Exato de Fisher, t-Student e Teste de Mann Whitney. Resultados: as voluntárias de meia idade apresentaram diferença significativa no domínio consequências negativas $(p=0,0121)$, além disso, atribuíram um número maior de doenças ao envelhecimento, o que sugere uma percepção mais negativa do envelhecimento neste grupo. A maioria das voluntárias associou procedimentos estéticos à melhora na saúde $(88,6 \%)$ mas sem diferença estatística significativa entre os grupos. Conclusão: Nos grupos estudados, os resultados sugerem uma percepção mais negativa do envelhecimento entre as mulheres de meia idade. A maioria das voluntárias relacionou os tratamentos estéticos com melhora na saúde.
\end{abstract}

Palavras chave: Meia idade. Idosas. Envelhecimento. Percepção. Estética.

\section{Perception of aging: middle-aged and elderly women who seek by aesthetic procedures}

\begin{abstract}
This study had as its objective to analyze whether there is significant statistical difference regarding the perception of aging among middle-aged women and elderly women that seek aesthetic procedures and in relation to perception of health improvement by performing aesthetic procedures in this same group. Method: Clinical, pilot, observational and cross-sectional study conducted with 70 women from 40 to 75 years old, used to aesthetic treatments in the city of São Paulo (SP). The instruments used were: validated Aging Perception Questionnaire and socio-demographic and description of participants questionnaires. The Chi-square, Fisher's exact, t-Student and Mann Whitney tests were used for data analysis. Results: The middle-aged volunteers presented significant difference in the domain negative consequences $(p=0.0121)$, moreover, attributed a greater number of diseases to aging, suggesting a more negative perception of aging in this Group. Most volunteers associated aesthetic procedures to health improvement (88.6\%), but with no significant difference between groups. Conclusion: In the studied groups, the results suggest a more negative perception of aging among middle-aged women. Most volunteers relate aesthetic treatments with health improvement.
\end{abstract}

Keywords: Middle age. Elderly. Aging. Perception. Aesthetics.

\footnotetext{
1 Pós graduação em Gestão em Cosmetologia pela Associação Brasileira de Cosmetologia. Mestrado em Ciências da Saúde pela UNISA. flaviafcarrara@gmail.com;

${ }^{2}$ Doutorado em Ciências Biológicas (Bioquímica) pela Universidade de São Paulo. Departamento: Pós graduação em Ciências da Saúde UNISA. E-mail: carmchris@yahoo.com;

${ }^{3}$ Doutorado em Enfermagem pela Escola de Enfermagem da Universidade de São Paulo . Departamento: Pós graduação em Ciências da Saúde - UNISA. E-mail: 1lpereira@unisa.br.
} 


\section{Introdução}

A percepção do envelhecimento engloba facetas como a satisfação com o envelhecimento e a idade subjetiva (capacidade do indivíduo mais velho se perceber ou se avaliar como alguém mais jovem). Idades subjetivas mais jovens e elevada satisfação com o envelhecimento podem ser indicadores da capacidade dos mais velhos para se adaptarem a perdas relacionadas com o envelhecimento. Além disso, indivíduos com uma percepção mais positiva do envelhecimento são menos vulneráveis aos estereótipos negativos ligados às pessoas mais velhas (SNEED, 2005; INGRAND et al., 2018).

A percepção do envelhecimento parece ser um bom indicador de um envelhecimento bem sucedido e tem sido sugerida como um preditor da capacidade funcional e da longevidade na velhice, tendo relação com aspectos do equilíbrio psicológico como a satisfação e a qualidade de vida, a solidão e a depressão. Quanto melhor a saúde de um indivíduo, percebida por ele mesmo, melhor sua percepção de envelhecimento (BARKER et al., 2007; YASSINE, 2011; RAMOS et al., 2012).

A maneira como as mulheres enfrentam seu processo de envelhecimento e a sua própria velhice dependem, além dos aspectos individuais, de um conjunto de fatores advindos da realidade social, econômica e cultural. As mulheres apresentam maior insatisfação com a imagem corporal e são mais sensíveis às influências sociais em relação à aparência quando comparadas aos homens. Assim, sofrem maior impacto em relação à visibilidade do envelhecimento, e antecipam as fases da adolescência e adulta , prorrogando o início da fase da velhice (DURIDAN et al., 2014; FIN et al., 2015; TORRES et al., 2015; AGUIAR et al., 2018).

A preocupação da mulher em envelhecer com saúde e beleza levou a um aumento da procura por tratamentos estéticos rejuvenescedores, por isso as indústrias inserem no mercado produtos, técnicas e aparelhos específicos direcionados principalmente ao público feminino, como alternativa para o resgate da juventude. As práticas de rejuvenescimento estão relacionadas a maior autonomia e capacidade funcional, visando também benefícios à saúde e bem estar, por isso têm caráter de autocuidado (WITCZAK et al., 2013; OLIVEIRA et al., 2015; CASTRO et al., 2016; MORANDO et al., 2017; CASTRO et al., 2018). 
Mulheres mais velhas podem experimentar menos insatisfação com o corpo devido a sua maturidade, experiências e autoestima positiva. A estética toma um novo sentido e a beleza na velhice significa saúde e cuidado de si e de suas relações (FIN et al., 2017).

Considerando o climatério (período de transição entre os anos reprodutivos e não reprodutivos da mulher) como fase determinante no processo de envelhecimento e tendo este início aproximado aos 40 anos de idade, segundo o Ministério da Saúde, considera-se neste trabalho mulheres de meia idade aquelas com 40 a 59 anos de idade. Nesta pesquisa, adotou-se também a definição de idoso da OMS para países em desenvolvimento (idosa é aquela pessoa com 60 anos de idade ou mais) (FEBRASGO, 2010; VALENÇA et al., 2010; ANTUNES; SILVA, 2013).

O estudo pretende fornecer dados para a melhor compreensão da visão sobre o processo de envelhecimento analisando a forma como é interpretado por mulheres de meia idade e mulheres idosas, contribuindo para o conhecimento mais aprofundado deste grupo e dando embasamento para uma avaliação mais assertiva de suas necessidades.

Esta pesquisa teve como objetivos analisar se há diferença estatística significativa relativa à percepção do envelhecimento entre mulheres de meia idade e mulheres idosas que buscam por procedimentos estéticos e em relação à percepção da melhora da saúde com a realização de procedimentos estéticos.

\section{Método}

Estudo clínico, piloto, observacional e transversal realizado com 70 mulheres de 40 anos a 75 anos de idade, adeptas de procedimentos estéticos, na cidade de São Paulo (SP), Brasil. A cidade de São Paulo possui atualmente 1,7 milhões de idosos no município, correspondendo a $15 \%$ da população paulistana. O envelhecimento no município está acima da média nacional e é constituído, sobretudo, por mulheres. Em 2017, 60\% dos idosos eram desse gênero. As mulheres totalizam 52,7\% da população da cidade. Antes uma metrópole industrial, agora São Paulo tem no setor de serviços o mais representativo correspondendo a 73,2\% do PIB estadual (PREFEITURA MUNICIPAL DE SÃO PAULO, 2019).

A amostra foi representada por clientes frequentadoras de espaços de estética e beleza recrutadas pelas esteticistas a quem a pesquisadora presta consultoria na área. As entrevistas

40 Id on Line Rev. Mult. Psic. V.14, N. 49 p. 38-50, Fevereiro/2020 - ISSN 1981-1179 Edição eletrônica em http://idonline.emnuvens.com.br/id 
foram agendadas nos locais de trabalho das profissionais de estética e foram aplicadas pela própria pesquisadora no período de outubro de 2016 a junho de 2017. Como não há estudos prévios com informações a respeito do desfecho principal em população semelhante, realizamos um estudo piloto sem cálculo de tamanho da amostra.

Os critérios de inclusão foram mulheres com idade entre 40 a 75 anos de idade, adeptas de tratamentos estéticos faciais ou corporais. Os critérios de exclusão foram mulheres não alfabetizadas ou incapacitadas de ler, entender e responder os questionários. Foram aplicados questionários que possibilitaram conhecer o perfil das voluntárias com questões relativas à idade, renda, grau de escolaridade, estado marital, procedimentos estéticos realizados, assim como, a aplicação do Questionário de Percepção do Envelhecimento validado no Brasil (FERREIRA et al., 2014; ABEP, 2016).

O Questionário de Percepção do Envelhecimento ou Aging Perception Questionnaire (APQ) é um instrumento multidimensional profundamente conectado com a percepção de saúde e foi desenhado para avaliar a autoadaptação ao contexto do envelhecimento. Esse questionário avalia a percepção do envelhecimento a partir de oito domínios distintos, que envolvem opiniões sobre o próprio envelhecimento e examinam a experiência dos indivíduos com as alterações em relação às doenças (RAMOS et al., 2012; FERREIRA et al., 2014).

A primeira parte do APQ é composta por 23 itens e avalia a opinião do indivíduo sobre seu envelhecimento através de sete domínios: Cronológico crônico e Cronológico cíclico (relacionados a duração dos eventos do envelhecimento), Consequências positivas e, Consequências negativas (avaliam os impactos positivos e negativos dos eventos relacionados ao envelhecimento), Controle positivo e Controle negativo (avaliam o controle sobre os eventos relacionados ao envelhecimento) e Representações Emocionais (avalia as respostas emocionais aos eventos relacionados ao envelhecimento). Com escala tipo Likert os escores mais elevados para cada domínio indicam maior aprovação de uma percepção específica. A segunda parte, com 17 itens, avalia a existência de doenças e sua relação com o envelhecimento através do domínio identidade, onde as respostas possíveis são sim ou não. Para as respostas sim os participantes são questionados se relacionam essas doenças com o fato de estarem envelhecendo, sendo sim ou não as respostas. O APQ apresentou boas propriedades psicométricas e foi considerado um instrumento adequado para avaliar a percepção do envelhecimento no Brasil (RAMOS et al., 2012; FERREIRA et al., 2014). 
As variáveis categóricas (escolaridade, estrato sócio econômico, estado marital) foram apresentadas com frequências absolutas e relativas. As respostas da parte B do APQ foram avaliadas com o Teste Qui-quadrado ou Teste Exato de Fisher (SIEGEL; CASTELLAN Jr, 2006). As médias de idade entre os grupos (meia idade e idosas) foram comparadas com o Teste t-Student (SIEGEL; CASTELLAN Jr, 2006) e os escores das questões da parte A do questionário APQ foram apresentados como mediana e intervalo interquartílico e comparadas com o Teste de Mann-Whitney (SIEGEL; CASTELLAN Jr, 2006). Os valores de $p<0,05$ foram considerados significativos.

A pesquisa foi aprovada pelo Comitê de Ética e Pesquisa da UNISA sob parecer número 1.673.934 e todas as voluntárias assinaram o Termo de Consentimento Livre e Esclarecido.

\section{Resultados}

A caracterização das voluntárias encontra-se na Tabela 1, segundo os grupos de meia idade e idosas. O grupo de voluntárias de meia idade tem proporção maior de nível superior de escolaridade. Os grupos são homogêneos em relação ao estrato sócio econômico e estado marital.

Tabela 1 - Caracterização das participantes segundo os grupos de meia idade e idosas (N= 70). São Paulo (SP), 2017.

\begin{tabular}{lccc}
\hline Variável & $\begin{array}{c}\text { Meia idade } \\
\mathrm{n}=51\end{array}$ & $\begin{array}{c}\text { Idosas } \\
\mathrm{n}=19\end{array}$ & $P$ \\
\hline Idade (anos), média e desvio padrão & $46 \pm 6$ & $64 \pm 4$ & $<0,0001^{*}$ \\
\hline Escolaridade , n (\%) & & & $0,0131^{* *}$ \\
$\quad$ Médio & $5(9,8 \%)$ & $7(36,8 \%)$ & \\
$\quad$ Superior & $46(90,2 \%)$ & $12(63,2 \%)$ & \\
\hline Estrato sócio econômico, n (\%) & & $0,2445^{* * *}$ \\
A & $26(54,2 \%)$ & $6(33,3 \%)$ & \\
B1 & $12(25,0 \%)$ & $8(44,4 \%)$ & \\
B2 & $10(20,8 \%)$ & $4(22,2 \%)$ & \\
\hline Estado marital, n (\%) & & & $0,5049^{* *}$ \\
Com companheiro & $42(82,4 \%)$ & $14(73,7 \%)$ & \\
Sem companheiro & $9(17,6 \%)$ & $5(26,3 \%)$ & \\
\hline
\end{tabular}

*Teste t-Student; ${ }^{* *}$ Teste Exato de Fisher; ${ }^{* * *}$ Teste Qui-quadrado 
Os resultados do domínio consequências negativas (Tabela 2) mostraram que o grupo de mulheres com meia idade apresentaram escores maiores no item 13 (Envelhecer torna tudo mais difícil para mim) do que o grupo de idosas. Não se observou significâncias estatísticas nos demais domínios da parte A do APQ.

Tabela 2 - Percepção do envelhecimento por parte de mulheres dos grupos de meia idade e idosas $(\mathrm{N}=70)$, conforme as respostas da subescala domínio consequências negativas do APQ (Aging Perception Questionnaire). São Paulo (SP), 2017.

\begin{tabular}{|c|c|c|c|}
\hline $\begin{array}{l}\text { ITEM } \\
\text { Mediana (intervalo interquartílico) }\end{array}$ & $\begin{array}{l}\text { MEIA IDADE } \\
\mathrm{n}=51\end{array}$ & $\begin{array}{c}\text { IDOSAS } \\
n=19\end{array}$ & $p^{*}$ \\
\hline \multicolumn{4}{|l|}{ Domínio consequências negativas do APQ } \\
\hline $\begin{array}{l}\text { 12- Envelhecer limita as coisas que posso } \\
\text { fazer }\end{array}$ & $3(2-4)$ & $2(2-4)$ & 0,1893 \\
\hline $\begin{array}{l}\text { 13- Envelhecer torna tudo mais difícil para } \\
\text { mim }\end{array}$ & $4(3-4)$ & $3(2-4)$ & 0,0121 \\
\hline $\begin{array}{l}\text { 14- À medida que envelheço, consigo } \\
\text { participar de menos atividades }\end{array}$ & $4(2-4)$ & $2(2-4)$ & 0,2286 \\
\hline
\end{tabular}

*Teste não paramétrico de Mann-Whitney

APQ: Aging Perception Questionnaire.

No domínio identidade do APQ (Tabela 3), ansiedade e problemas com sono foram mais frequentes nas mulheres de meia idade. No grupo de idosas a diminuição de ritmo e problemas nos pés foram as alterações mais frequentes. $\mathrm{O}$ grupo de mulheres de meia idade apresentou percentual maior em alteração de sono e ansiedade em relação ao grupo de idosas, respectivamente. Entretanto, o quesito problema nos pés foi relatado com mais frequência no grupo de idosas em relação ao grupo de meia idade.

Tabela 3 - Alterações/mudanças na saúde da subescala domínio identidade (parte B) do APQ (Aging Perception Questionnaire) segundo o grupo de mulheres de meia idade e idosas (N=70). São Paulo (SP), 2017.

\begin{tabular}{lccc}
\hline Alterações & $\begin{array}{c}\text { Meia idade } \\
\mathrm{n}=51\end{array}$ & $\begin{array}{c}\text { Idosas } \\
\mathrm{n}=19\end{array}$ & $P$ \\
\hline Peso & $40(78,4 \%)$ & $14(73,7 \%)$ & $0,7519^{* * *}$ \\
Problemas com sono & $25(49,0 \%)$ & $4(21,1 \%)$ & $0,0347^{* * * *}$ \\
Problemas costas/hérnias & $26(51,0 \%)$ & $11(57,9 \%)$ & $0,6063^{* * *}$ \\
Dores articulações & $18(35,3 \%)$ & $9(47,4 \%)$ & $0,3561^{* * *}$ \\
Perda de mobilidade & $11(21,6 \%)$ & $3(15,8 \%)$ & $0,7437^{* *}$
\end{tabular}


Perda de equilíbrio

Perda de força

Diminuição de ritmo

Câimbras

Problemas ossos/articulações

Problemas cardíacos

Problemas de audição

Alterações na visão

Problemas respiratórios

Problemas nos pés

Depressão

Ansiedade
$7(13,7 \%)$

$11(21,6 \%)$

$22(43,1 \%)$

$12(23,5 \%)$

$16(31,4 \%)$

$5(9,8 \%)$

$4(7,8 \%)$

$37(72,5 \%)$

$6(11,8 \%)$

$8(15,7 \%)$

$17(33,3 \%)$

$35(68,6 \%)$
$2(10,5 \%) \quad 1,0000^{* *}$

$5(26,3 \%) \quad 0,7519^{* *}$

$9(47,4 \%) \quad 0,7917^{* * *}$

$5(26,3 \%) \quad 1,0000^{* * *}$

$10(52,6 \%) \quad 0,1016^{* * *}$

$2(10,5 \%) \quad 1,0000^{* *}$

$1(5,3 \%) \quad 1,0000^{* *}$

$12(63,2 \%) \quad 0,4458^{* * *}$

$1(5,3 \%) \quad 0,6649^{* *}$

$8(42,1 \%) \quad 0,0274^{* *}$

$2(10,5 \%) \quad 0,0564^{* * * *}$

$6(31,6 \%) \quad 0,0051^{* * *}$

*** Teste Exato de Fisher; ${ }^{* * *}$ Teste Qui-quadrado.

Quando as participantes do estudo foram questionadas se relacionavam as alterações de saúde somente com o fato de estarem envelhecendo (Tabela 4), no grupo de meia idade as alterações com frequências maiores de associação com envelhecimento foram alterações na visão, problemas de audição e diminuição de ritmo. Já nas idosas foram problemas com sono, diminuição de ritmo e problemas nos ossos e articulações. As pacientes de meia idade com problemas com sono relatam uma proporção menor de associação desta alteração com o processo de envelhecimento em relação às idosas. Ainda na Tabela 4, nota-se que as mulheres de meia idade associaram todas as doenças ao envelhecimento (com exceção do item depressão).

Tabela 4 - Associação das Alterações/mudanças na saúde com o processo do envelhecimento da subescala domínio identidade (parte B) do APQ (Aging Perception Questionnaire) segundo o grupo de mulheres de meia idade e idosas (N=70). São Paulo (SP), 2017.

\begin{tabular}{lccc}
\hline Alterações & $\begin{array}{c}\text { MEIA IDADE } \\
\mathrm{n}=51\end{array}$ & $\begin{array}{c}\text { IDOSAS } \\
\mathrm{n}=19\end{array}$ & $P$ \\
\hline Peso & $19 / 40(47,5 \%)$ & $7 / 14(50,0 \%)$ & $0,8720^{* * *}$ \\
Problemas com sono & $9 / 25(36,0 \%)$ & $4 / 4(100 \%)$ & $0,0301^{* * *}$ \\
Problemas costas/hérnias & $11 / 26(42,3 \%)$ & $5 / 11(45,5 \%)$ & $1,0000^{* *}$ \\
Dores articulações & $9 / 18(50,0 \%)$ & $5 / 9(55,6 \%)$ & $1,0000^{* *}$ \\
Perda de mobilidade & $7 / 11(63,6 \%)$ & $2 / 3(66,7 \%)$ & $1,0000^{* *}$ \\
Perda de equilíbrio & $3 / 7(42,9 \%)$ & $0 / 2(0 \%)$ & $0,5000^{* *}$ \\
Perda de força & $7 / 11(63,6 \%)$ & $3 / 5(60,0 \%)$ & $1,0000^{* *}$ \\
Diminuição de ritmo & $15 / 22(68,2 \%)$ & $9 / 9(100 \%)$ & $0,0766^{* *}$
\end{tabular}




\begin{tabular}{lccc} 
Câimbras & $2 / 12(16,7 \%)$ & $0 / 5(0 \%)$ & $1,0000^{* *}$ \\
Problemas ossos/articulações & $10 / 16(62,5 \%)$ & $8 / 10(80,0 \%)$ & $0,4198^{* *}$ \\
Problemas cardíacos & $2 / 5(40,0 \%)$ & $1 / 2(50,0 \%)$ & $1,0000^{* *}$ \\
Problemas de audição & $3 / 4(75,0 \%)$ & $0 / 1(0 \%)$ & $0,4000^{* *}$ \\
Alterações na visão & $30 / 37(81,1 \%)$ & $9 / 12(75,0 \%)$ & $0,6497^{* * *}$ \\
Problemas respiratórios & $1 / 6(16,7 \%)$ & $0 / 1(0 \%)$ & $1,0000^{* *}$ \\
Problemas nos pés & $3 / 8(37,5 \%)$ & $5 / 8(62,5 \%)$ & $0,6193^{* *}$ \\
Depressão & $0 / 17(0 \%)$ & $1 / 2(50 \%)$ & $0,1053^{* *}$ \\
Ansiedade & $5 / 35(14,3 \%)$ & $0 / 6(0 \%)$ & $1,0000^{* *}$ \\
\hline
\end{tabular}

*** Teste Exato de Fisher; ${ }^{* * *}$ Teste Qui-quadrado.

A Tabela 5 mostra que a maioria das mulheres de meia idade e das mulheres idosas responderam que relacionavam procedimentos estéticos à melhora na saúde. No entanto, não houve diferença estatisticamente significativa entre os grupos.

Tabela 5 - Distribuição das mulheres dos grupos de meia idade ou idosas conforme o relato do relacionamento dos procedimentos estéticos à melhora na saúde $(\mathrm{N}=70)$. São Paulo $(\mathrm{SP})$, 2017.

\begin{tabular}{lccc}
\hline Variável & $\begin{array}{c}\text { Meia idade } \\
\mathrm{n}=51\end{array}$ & $\begin{array}{c}\text { Idosas } \\
\mathrm{n}=19\end{array}$ & $p^{*}$ \\
\hline Relaciona os procedimentos estéticos & & & 0,6738 \\
à melhora na saúde & & & \\
Não & $5(9,8 \%)$ & $3(15,8 \%)$ & \\
Sim & $46(90,2 \%)$ & $16(84,2 \%)$ & \\
\hline
\end{tabular}

*Teste Exato de Fisher

\section{Discussão}

Os resultados sugerem que as mulheres de meia idade possuem percepção mais negativa sobre a experiência de envelhecer do que as mulheres idosas. Além disso, no domínio identidade, atribuíram um número maior de alterações na saúde ao processo do envelhecimento, o que pode estar relacionado a crenças negativas sobre o envelhecimento.

Em domínio consequências negativas as mulheres de meia idade apresentaram escores maiores que as idosas. Há maior aprovação neste grupo para o fato de que "envelhecer torna tudo mais difícil” (item 13 do APQ). Para autores como Mori e Coelho (2003), alguns adultos 
se mostram incapazes de renunciar à juventude por temerem tarefas desconhecidas, sacrifícios e perdas associadas à velhice. As mulheres encaram o envelhecimento como uma fase de perda da autonomia pois este processo é relacionado ao declínio da saúde e a decadência (FERREIRA et al., 2013; MARI et al., 2016).

No domínio identidade, as mulheres de meia idade atribuíram todas as doenças ao envelhecimento em níveis diferentes (com exceção da depressão), sugerindo que estas mulheres associam doença e incapacidade com velhice. As idosas atribuíram um número menor de alterações na saúde ao envelhecimento. Para elas, as alterações ansiedade, problemas respiratórios, problemas de audição, câimbras e perda de equilíbrio não estão relacionadas somente ao processo de envelhecimento. Estes resultados contrariam os de Fernandes ${ }^{26}$, onde em estudo comparativo entre pessoas de meia idade e pessoas idosas um maior número de doenças foi atribuído ao envelhecimento no grupo dos idosos. Indivíduos com menos problemas de saúde estão menos conscientes do envelhecimento e tem menor oscilação sobre a ciência do processo (FERNANDES, 2014).

Os problemas com sono foram mais relatados entre as mulheres de meia idade. Porém, esse problema foi mais relacionado ao envelhecimento no grupo de idosas. O que pode explicar essa alteração entre as mulheres de meia idade é o próprio climatério que provoca entre outros sintomas, quadros de insônia e mudanças na qualidade do sono. Insônia ou sono agitado são sintomas neurovegetativos encontrados frequentemente durante o climatério, mas também podem estar associados a outras etiologias (BRASIL, 2008).

Parece haver um indício de que a depressão, nesta pesquisa, também é prevalente entre as mulheres de meia idade, quando comparadas as idosas. A depressão, porém, foi uma das alterações de saúde menos atribuídas ao envelhecimento, confirmando que outros fatores devem explicar a presença desta alteração. Barker ${ }^{5}$ associa em sua amostra as consequências negativas do envelhecimento a problemas funcionais e depressão, assim como a atitudes e expectativas mais negativas sobre o envelhecimento.

A ansiedade também foi mais relatada entre as mulheres de meia idade em comparação as idosas. Ansiedade e depressão, estão relacionadas nos trabalhos de Barker ${ }^{5}$ a idosos , contrariando os resultados da presente pesquisa. Ansiedade e depressão são possíveis sintomas do climatério, segundo o Manual de Atenção ao Climatério (BRASIL, 2008), podendo apresentar-se isoladamente ou em conjunto em algum período deste processo em intensidade 
variável. Freeman et al. (2016) afirmam que estudos sobre a relação da percepção negativa do envelhecimento com desordens mentais são escassos. Os mesmos autores relataram que no grupo estudado em sua pesquisa, a depressão e ansiedade são consequências da percepção negativa do envelhecimento. Entre aqueles que já possuem o problema a percepção negativa reforçou a persistência das condições.

Apesar de não haver diferença significativa entre os grupos, a maioria das voluntárias associaram os procedimentos estéticos à melhora na saúde. Essa informação remete a definição de saúde dada pela Organização Pan-americana de saúde: A saúde não é só a ausência de doença, mas abrange o bem-estar geral. Em pesquisa sobre a estética na qualidade de vida de idosos, $87 \%$ dos idosos participantes demonstraram realizar procedimentos estéticos para autocuidado, refletindo no bem-estar e autoestima e 36,7\% entendem que a estética influencia na qualidade de vida e cuidados com a saúde (BRASIL, 2005; CERVI, 2014).

Em nossas buscas não foram encontrados artigos, envolvendo a aplicação do APQ em amostras similares à utilizada nesta pesquisa. Assim, destaca-se a inovação desta pesquisa ao trazer resultados específicos para a população utilizada. Deve-se considerar também o fato de que as principais pesquisas utilizadas como referência nesta discussão foram realizadas em países europeus, onde a realidade e cultura, diferentes no Brasil, devem certamente influenciar nos resultados. Apesar disso, a amostra foi composta por mulheres com condições socioeconômicas elevadas para a média da população brasileira.

Uma limitação de nosso estudo diz respeito ao tamanho da amostra de idosas (menor que a de mulheres de meia idade). O número reduzido de participantes não permite extrair conclusões para a população de mulheres de meia idade e de mulheres idosas em geral.

\section{Conclusão}

Os resultados obtidos sugerem que neste estudo as mulheres de meia idade que buscaram por tratamentos estéticos apresentam uma percepção mais negativa do envelhecimento, apresentando escores mais altos no domínio relacionado às consequências negativas do processo. Além disso, atribuíram maior número de alterações na saúde ao 
envelhecimento, quando comparadas às mulheres idosas, mostrando que associaram doença à velhice.

Apesar de a maioria das voluntárias relacionarem os tratamentos estéticos com a melhora na saúde, não houve diferença significativa nos resultados entre os grupos.

Para futuras pesquisas, sugere-se realizar estudo com uma amostra mais equilibrada de mulheres idosas ou um estudo longitudinal que avaliasse o percurso destas mulheres no decorrer do tempo, acompanhando a transição das mulheres de meia idade em mulheres idosas.

Os resultados do presente estudo, direcionados aos pesquisadores do segmento de estética e envelhecimento, poderão contribuir para a melhor compreensão do processo do envelhecimento, nas diferentes faixas etárias entre mulheres e, consequentemente, nortear estratégias específicas de atendimento às mulheres e estimular novas pesquisas sobre o referido público.

\section{Referências}

ABEP - Associação Brasileira de Empresas de Pesquisa. Critério de classificação econômico Brasil. Atualização da distribuição de classes para 2016. Disponível em: www.abep.org

AGUIAR, A.; CAMARGO, B. V.; BOUSFIELD, A. B. S. Envelhecimento e prática de rejuvenescimento: Estudo de representações sociais. Psicologia: Ciência e Profissão, v. 38, n. 3, p. 494-506, 2018.

ANTUNES, P. C.; SILVA, A. M. Elementos sobre a concepção da Meia Idade, no processo de envelhecimento humano. Revista Kairós Gerontologia. São Paulo (SP), v. 16, n. 5, p. 123-40, 2013.

BARKER, M., et al. Cross-sectional validation of the Aging Perceptions Questionnaire: a multidimensional instrument for assessing self-perceptions of aging. BMC Geriatr., v. 7, p. 9, 2007.

BRASIL. Ministério da Saúde. Secretaria de Atenção à Saúde. Departamento de Ações Programáticas Estratégicas. Manual de Atenção à Mulher no Climatério/Menopausa. Brasília: Editora do Ministério da Saúde, 2008. 192 p. (Série A. Normas e Manuais Técnicos) (Série Direitos Sexuais e Direitos Reprodutivos - Caderno n. 9).

BRASIL. Organização Pan-Americana da Saúde. Envelhecimento ativo: uma política de saúde. Brasília-DF: Organização Pan-Americana da Saúde, 2005.

CASTRO, A., et al. Representações sociais do envelhecimento e do rejuvenescimento para mulheres que adotam práticas de rejuvenescimento. PSICO - Porto Alegre, v. 47, n. 4, p. 319-30, 2016.

48 Id on Line Rev. Mult. Psic. V.14, N. 49 p. 38-50, Fevereiro/2020 - ISSN 1981-1179

Edição eletrônica em http://idonline.emnuvens.com.br/id 
CASTRO, A.; GIACOMOZZI, A. I.; CAMARGO, B. V. Representações sociais, zona muda e práticas sociais femininas sobre envelhecimento e rejuvenescimento. Est. Inter. Psicol., Londrina, v. 9, n. 2, p. 58-77, 2018.

CERVI, C. R. Estética na qualidade de vida de idosos [Dissertação de Mestrado]. Porto Alegre: Pontifícia Universidade Católica do Rio Grande do Sul, 2014.

DURIDAN, A.; SANTOS, D. F.; GATTI, A. L. Autoestima e cuidados pessoais em mulheres de 60 a 75 anos. Aletheia, v. 43-44, p. 174-87, 2014.

FEDERAÇÃO BRASILEIRA DAS ASSOCIAÇÕES E GINECOLOGIA E OBSTETRÍCIA (FEBRASGO). Climatério: manual de orientação. São Paulo: FEBRASGO; 2010.

FERNANDES, A. F. R. A auto-percepção do envelhecimento e o bem-estar psicológico [Dissertação de Mestrado Integrado em Psicologia]. Lisboa, Portugal: Faculdade de Psicologia, Universidade de Lisboa, 2014.

FERREIRA, L.; NEVES, A. N.; TAVARES, M. C. G. C. F. Validity of body image scales for Brazilian older adults. Motriz: Rev. Educ. Fis., v 20, n. 4, p. 359-73, 2014.

FERREIRA, V. N. et al. Menopausa: marco biopsicossocial do envelhecimento feminine. Psicologia \& Sociedade, v. 25, n. 2, p. 410-9, 2013.

FIN, T. C., et al. Estética e expectativas sociais: o posicionamento da mulher idosa sobre os recursos estéticos. Revista Kairós Gerontologia, São Paulo (SP), Brasil, v. 18, n. 4, p. 133-149, 2015.

FIN, T. C.; PORTELLA, M. R.; SCORTEGAGNA, S. A. Velhice e beleza corporal das idosas: conversa entre mulheres. Rev. Bras. Geriatr. Gerontol., Rio de Janeiro, v. 20, n. 1, p. 77-87. 2017.

FREEMAN, A. T. et al. Negative perceptions of ageing predict the onset and persistence of depression and anxiety: Findings from a prospective analysis of the Irish Longitudinal Study on Ageing (TILDA). J. Affect. Disord., v. 199, p. 132-8, 2016.

INGRAND, I. et al. Positive perception of aging is a key predictor of quality-of-life in aging people. PLoS One, v. 13, n. 10, p. e0204044, 2018.

MARI, F. R.; ALVES, G. G.; AERTS, D. R. G. S.; CAMARA, S. O processo de envelhecimento e a saúde : o que pensam as pessoas de meia idade sobre o tema. Rev. Bras. Geriatr. Gerontol., v. 19, n. 1, p. 35-44, 2016.

MORANDO, E.; SCHMITT, J.; FERREIRA, M. Envelhecimento, autocuidado e memória: intervenção como estratégia de prevenção. Revista Kairós: Gerontologia, v. 20, n. 2, p. 353-74, 2017.

MORI, M. E.; COELHO, V. L. D. A vida ouvida: a escuta psicológica e a saúde da mulher de meia idade. Estudos e Pesquisas em Psicologia, v. 3, n. 2, p. 36-78, 2003.

OLIVEIRA, E. F. S.; MERCADANTE, E, F.; PORTO, E. F. Percepção de homens e mulheres maiores de 50 anos sobre a estética do envelhecimento. LifeStyle Journal, São Paulo, v. 2, n. 2, p. 83-98, 2015.

49 Id on Line Rev. Mult. Psic. V.14, N. 49 p. 38-50, Fevereiro/2020 - ISSN 1981-1179

Edição eletrônica em http://idonline.emnuvens.com.br/id 
PREFEITURA MUNICIPAL DE SÃO PAULO, Dados estatísticos de São Paulo, 2019, disponível em:

https://www.prefeitura.sp.gov.br/cidade/secretarias/urbanismo/dados_estatisticos/informes_urban $\mathrm{os} / \mathrm{p}=273565$

RAMOS, L. M. B. C. et al. Tradução e adaptação cultural do APQ-Aging Perceptions Questionnaire para a língua portuguesa brasileira. Rev. Bras. Geriatr. Gerontol., Rio de Janeiro, v. 15, n. 2, p. 233 42, 2012.

SIEGEL, S.; CASTELLAN Jr, N. J. Estatística não paramétrica para ciências do comportamento. $2^{\mathrm{a}}$ ed. Porto Alegre: Artmed, 2006.

SNEED, J. R.; WHITBOURNE, S. Models of the aging self. Journal of Social Issues, v. 61, n. 2, p. 375-88, 2005.

TORRES, T. L. et al. Representações sociais e crenças normativas sobre envelhecimento. Ciência \& Saúde Coletiva, v. 20, n. 12, p. 3621-30, 2015.

VALENÇA, C. N.; NASCIMENTO FILHO, J. M.; GERMANO, R. M. Mulher no climatério: reflexões sobre desejo sexual, beleza e feminilidade. Saúde Soc. São Paulo, v. 19, n. 2, p. 273-85, 2010.

WITCZAK, P. E.; ZAMBERLAN, L.; SPAREMBERGER, A. O envelhecer e a beleza feminina: significados e comportamentos de consumidoras de produtos associados. In: XXXIII Encontro Nacional de Engenharia de Produção. A Gestão dos Processos de Produção e as Parcerias Globais para o Desenvolvimento Sustentável dos Sistemas Produtivos. Salvador, BA, Brasil, 08 a 11 de outubro de 2013.

YASSINE, I. M. C. A auto-percepção do envelhecimento e os traços de personalidade em idosos. [Dissertação Mestrado Integrado em Psicologia]. Lisboa: Faculdade de Psicologia, Universidade de Lisboa, 2011.

\section{Como citar este artigo (Formato ABNT):}

CARRARA, Flávia Franco; VINAGRE, Carmen Guilherme Christiano de Matos; PEREIRA, Luciane Lúcio. Percepção do envelhecimento: mulheres de meia idade e idosas que buscam por procedimentos estético. Id on Line Rev.Mult. Psic., Fevereiro/2020, vol.14, n.49, p. 38-50 . ISSN: 1981-1179.

Recebido: 03/12/2019;

Aceito: 08/01/2020 